\title{
Graphic User Interface Design Principles for Designing Augmented Reality Applications
}

\author{
Afshan Ejaz ${ }^{1}$, Dr Syed Asim Ali ${ }^{2}$, Muhammad Yasir Ejaz ${ }^{3}$, Dr Farhan Ahmed Siddiqui ${ }^{4}$ \\ Department of Computer Science, Institute of Business Administration, Karachi, Pakistan ${ }^{1}$ \\ Department of Computer Science, FAST National University, Karachi, Pakistan ${ }^{3}$ \\ Department of Computer Science, UBIT - University of Karachi, Karachi, Pakistan ${ }^{2,4}$
}

\begin{abstract}
The reality is a combination of perception, reconstruction, and interaction. Augmented Reality is the advancement that layer over consistent everyday existence which includes content based interface, voice-based interfaces, voicebased interface and guide based or gesture-based interfaces, so designing augmented reality application interfaces is a difficult task for the maker. Designing a user interface which is not only easy to use and easy to learn but its more interactive and selfexplanatory which have high perceived affordability, perceived usefulness, consistency and high discoverability so that the user could easily recognized and understand the design. For this purpose, a lot of interface design principles such as learnability, Affordance, Simplicity, Memorability, Feedback, Visibility, Flexibly and others are introduced but there no such principles which explain the most appropriate interface design principles for designing an Augmented Reality application interfaces. Therefore, the basic goal of introducing design principles for Augmented Reality application interfaces is to match the user efforts and the computer display ("plot user input onto computer output") using an appropriate interface action symbol ("metaphors") or to make that application easy to use, easy to understand and easy to discover. In this study by observing augmented reality system and interfaces, few of well-known design principle related to GUI ("user-centered design") are identified and through them, few issues are shown which can be determined through the design principles. With the help of multiple studies, our study suggests different interface design principles which make designing Augmented Reality application interface more easier and more helpful for the maker as these principles make the interface more interactive, learnable and more usable. To accomplish and test our finding, Pokémon Go, an Augmented Reality game, was selected and all the suggested principles are implemented and tested on its interface. From the results, our study concludes that our identified principles are most important principles while developing and testing any Augmented Reality application interface.
\end{abstract}

Keywords-GUI; augmented reality; metaphors; affordance; perception; satisfaction; cognitive burden

\section{INTRODUCTION}

Perception, interaction and renovation are combination to form reality Augmented Reality is the modernization that cat over regular daily existence that include voice-based interfaces, map-based interfaces, text-based interface and gestures based interface so designing such application is quite difficult task for the designer. To make the Augmented Reality interface much easier and interactive, some design principles are introduced. To match the user efforts and the computer presentation using a suitable interface action symbol or to make that application easy to used, easy to discover and easy to understand, the design principles are introduce. The basic components to consider are as follows: interface physical part, the virtual graphic and sound-related demonstration and to associate all these metaphors related to interaction are used together. Fig. 1 shows the connection among all three components. The designer of the interface has available a wide combination of information and yield gadgets and technique for mapping contribution to yield. The test is to unite these together in a way that is most appropriate to the preferred job, energizes ease of use and gives an abnormal state of user execution and fulfillment.

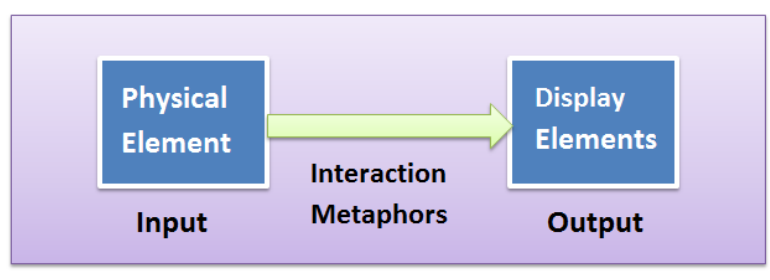

Fig. 1. The Key Interface Elements.

\section{LITERATURE REVIEW}

In the previous works of AR, Gabbard [1] has recognized design principles from broad gatherings. Specialists have used these rules, by being inspired from issues relating to specific designs for the AR framework. Although a subject of discussion, if particular design rules get to travel with respect to other AR interfaces. Therefore, the major goal is to develop common design rules or principles through the information available in the previous research. General HCI standards are taken as an approach and perceived on how AR frameworks improvements can be made by applying them or how they as of now have been connected [2]. This might bring about moderately expansive and general recommendations yet can serve as a beginning stage. Further refining can be done in particular issues and assignments. The beneath research is, in this way, an endeavor to talk about the advancement of this yet to be commonly undeveloped field, in the path of design rules. While creating rules for attempting to apply on augmented reality system, we should not only consider design principles of GUI but also consider some method of evaluation of GUI. Some significant contrasts amid the previous design of GUI and AR based interfaces. GUI design rules by and large recommend that the users are interacting with a PC screen, console and mouse. AR regularly 
consolidates different methods for communicating with the interface. Accordingly, there are conceivably extraordinary interaction potential outcomes that must be thought about. Be that as it may, utilization of information base of all-purpose Human Computer Interaction.

Moore [3] creates an effort to check the usability of a tangible AR interfaces by using Neilson listed heuristics [4]. $\mathrm{He}$ found that, while general and unclear, heuristics identify the task that needs to improve immediately [5]. When developing design rules or principles for AR we can likewise utilize learning realized by Virtual Reality (VR) writing. Contrasted with AR, inside VR explore, push to coordinate $\mathrm{HCI}$ related issues into innovation improvement has increased. Though a few frameworks of AR and VR share some elements from an interface and collaboration viewpoint, some likewise contrasts and specialists ought to contemplate over the particular one of a kind issues and necessities of $\mathrm{AR}$ frameworks.

An explanation behind user-centered design standards or principles being to a great extent neglected might be that there still is extremely restricted information here and not very many plan rules have been created. Most rules are fairly particular discoveries by scientists. One issue of creating rules or measure ease of use is immeasurable quantity of various AR frameworks and Input and output devices that have been utilized. Ranging from cell phones like mobile phones, PDAs show (HMD) established inside and open air frameworks, or substantially settled screen frameworks $[7,8]$. What's more, the constraint is not just restricted to visual interfaces but rather likewise may once in a while incorporate sound and haptic interfaces. Acknowledgment of UIs and the fundamental cooperation strategies turns out to be a somewhat difficult part when building up a framework on AR [9]. The AR space has not yet characterized its particular interface (and it is faulty in the event that it ever will). While AR UIs are normally acknowledged with an extensive assortment of communication systems and connection gadgets, a large portion of them rely on upon particular equipment [17].

Though for Web-based applications it appears slightly feasible to discover collective tools and guidelines for design to support usability engineering in the process is somewhat problematic for similar AR applications. While assigning desktop PCs we could depend on comparable I/O devices and additional or fewer ordinary collaboration procedures. For suppose taking screen catches might be legitimate for dissecting route on sites. Since AR interfaces contain virtual data enlisted in 3D desktop assessment procedures usually aren't appropriate.

Additionally, utilization of option information devices creates new difficulties and requests. For instance, the thought of snaps must be reached out to the possibility of a user input. Rizzo et al. contend with nonattendance of a built-up plan and interface philosophy as risen for the 2D desktop layout throughout our most recent 30 years, we still are restricted to exploratory, experimentation method of a way to deal with 3D interface as well as its association outline [10]. The moderately quick changes in equipment capacities, device accessibility and the cost are extra impediments for inferring general outline recommendations.

\section{Design PRinciples for Augmented Reality APPLICATION}

From the previous research different number of design principles and usability principles and heuristic was found [3]. Examining every one of them with regards to augmented reality system would go past the breaking points of this current research. Our attempted to suggest few important principles which might be very beneficial in developing augmented reality interface and we also discuss how we beneficial to AR interface, for this have follow some guidelines and principles of HCI. The purpose is to give great instances, in what ways design principles can apply to AR interfaces as shown in Fig. 2:

\section{A. Affordance or Perceived Affordance}

Affordance refers to the relationship between the user interface and the underlying properties associated with it. AR system uses easily understandable objects as metaphors for the sole purpose that users are able to identify its use or function by just observing it, hence reducing the learning materials for the users before using the AR system. Let alone this, it is also noticed that due to the usage of TUI interface may cause the meaning to change, henceforth it is important to define the metaphors in the documentation for the users.

Furthermore, use of interaction metaphors as a result of a motion may help ease the communication between the user and the AR system. Use of motion, as a form of interaction, may help users understand the functionality of the metaphors. Let alone this implementation of this concept in the AR system may reduce the work of the system in conventional methods of interaction, for e.g. use of a pointing device may require the system to do constant remapping of function and action not only this the Direct 3D manipulation provides direct access to the user into the systems, this approach is used in 3D learning and construction environments [11].

Furthermore, perceived affordance is the way the user perceives as being possible based on how an object is presented or an object should naturally imply what actions it supports through its design and attributes. In AR, computerized enlargements can appear as simple information overlays or complex multi-dimensional images [14]. Field of view is a valuable land in AR encounters where each thing ought to have a reason. It must be significant from a user point of view to interact with an object the way its interaction is perceived. For example, in representing background, a model which is 3D could be ascended, transformed and deployed upon. Whereas on other side scaling an rotating model of car object does not make much sense.to cope up with this meaningful principle of affordance could bepermitted form 3D objects can be interrelated with and their belongings transformed while 'joined on' models cannot be. 


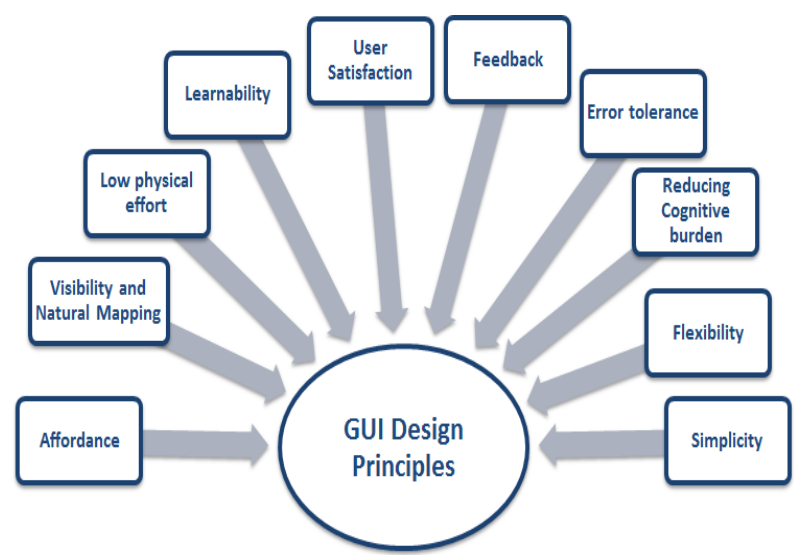

Fig. 2. Graphical user Interface Design Principles.

\section{B. Visibility and Natural Mapping}

There are few common pattern and standards which help human to interact with the object; the pattern will be natural or human derived pattern. Through these standards and pattern, we come to establishment of user's mental model means how user perceived thing how they used any object or perceived any object in interface. Here comes the concept of mapping comes; it is concept which associate components related to computer artifacts to this present reality. They are the connection between what you need to do and what is seen conceivable.it is the connection which map the concept of real to our virtual world. Great mappings are common and utilize physical analogies or social models. Therefore, they are seen promptly, simpler to recollect, and empower better convenience. A case of a poor mapping is that user can't be able to perceive how the object works $[12,15]$.

This is especially basic with AR frameworks or interfaces that are presenting totally new ideal models while existing with regards to the characteristic world. With these frameworks or interfaces, the comprehension of and adherence to characteristic human signals ("gestures") will be basic [13].

An efficient AR interface is one which map and integrate its reality to the real world. Doing as such will make the interface essentially an upgrade upon this present reality, rather than a simulated layer, It ought to be practically imperceptible. This is a troublesome test however it requires a comprehension of minimization of plan components, use of legitimate visual point of view, and maybe new visual strategies for mapping computerized symbolism onto this present reality. Hence, "static interface components" ought to be limited as they uphold the nearness of a counterfeit layer before the user's face $[10,16]$.

\section{Low Physical Effort}

Since the AR systems are being developed for the users, it should be kept in mind that the user should be able to achieve the task easily and since the interaction system may require motion from the user, it is advisable that the user-worn parts that are linked to the systems must be very comfortable and not put a strain on the body, which may diminish the success of the AR system if not planned well. AR systems may also cause the users to experience sickness due to certain environments and situation being exposed to the user, though this may not prove to be fatal in AR system, but when the user viewpoint may change from $A R$ representation to a VR representation, this transition between the two systems may also result in sickness and disorientation hence this point should be kept in mind before designing the AR application, let alone this the AR system should also inform the user of the usage time, due to the brawl between the Magic Leap and Microsoft over the safety of the newly introduced AR system by Microsoft, putting the argument of declarations apart, after an A Magic Leap spokesperson clarified Lebovitz's (CEO of Magic Leap) explains, proverb, "We believe if technology is not replicating all of the physiologic important parameters of a light-field, which the human to-neuro system requires, it can cause a spectrum of temporary and/or permanent neurologic deficits." Through this statement we can understand low usage time is advised for the users and since AR being not completely discovered may have hidden effects over the user and which we may not be able to observe yet.

\section{Learnability}

Learnability is related to how the user uses the system whether the user will be able to use the system easily by simply recognizing the system or whether the user should recall everything by memorizing it. Learnability problem of three-dimension UIs affect responses and deployment of framework or system by "regular" user deprived of earlier preparing with such innovation. Utilizing AR interface permits system developer to recognize novel collaboration systems that user has not experienced and connected yet. These might be not the same as how individuals would collaborate in and with genuine situations and issues. Through this way the user should learn to effectively a user can use the system. Instinctive communication systems and techniques that are likened to true conduct or like what the users as of now are utilized to can restrain the learning required [14].

After building and classifying interaction components, designers additionally ought to see self as a distinction. Kaufman restructured the menu structure of the augmented reality system and maps the components similar to the real menu component of shared desktop. This help the user to learn the system more easily as they used the similar system before as they are familiar with all the command and interaction step they provided. Design components and structures like this can enhance a frameworks' congruity with user desires and along these lines encourage learnability [16]. Additional elements which affect learnability is consistency (it is critical that the $\mathrm{UI}$ is predictable in existence and conduct).

Furthermore, Learnability is link with Consistency and Standards, as it is the major factor in the design principles, and if we talk about the consistency so gesture interaction is a major focus. There are no models as far as gestural interaction in AR. AR draws out a variety of practices from various users regarding how they explore a three-dimensional space. What is common for one is not normal for another. This creates a provocation in defining consistent gestures and interactions [17].

\section{E. User Satisfaction}

User satisfactory is the factor of design principles. The perceived user experience is an expansive component or factor 
and turns out to be more critical the nearer an AR framework draws in the user in exercises as opposed to resolving tasks. While developing the augmented reality interfaces, the usability of AR interfaces not only depend upon objectives dimensions but also focused on the subjective user perception. Information on these criteria can be assembled amid casual user testing, perceptions all through exhibits or formal lab assessments. Therefore, subjective and objective measures should be measured to understand the user satisfaction [12].

Furthermore, it also includes user perceived ability that does user will do what the user intended to do There are two kinds. False positives are activities that occur when I don't aim them to occur. For example," I don't want to move an object but it sticks to my hand and moves with it when my hand grazes by". False negatives are activities that don't occur when I aim it to occur. For example, "I try to unsuccessfully grab an object that is meant to be grabbed". This interaction is one of the most frustrating aspects of AR. This also relies on the underlying hardware and software algorithms working near flawlessly. There are some UX tricks one can play to improve the perceived reliability, but this remains a critical interaction.

\section{F. Feedback}

The failure to understand what going on the interface is one of the biggest drawbacks of the system when user is interacting with it. This is due to lack of feedback from the interface or system. Feedback Absence is related to status or progress or an error lead to user frustration. By providing simple feedback either in the form of graphics or textual form can convey about the status of the system about what happing or what the user have to do next. Keep in mind that users are not engineers, so precise specialized depiction is less critical than portraying the basic "main concern" ramifications of the status.

Furthermore, users simply tolerate a specific extent of system delay. For instance, if instructions which user will give to the system are not accomplished subsequently in a specific time, then it's difficult for the user to build the preserving model of reason and outcome. Through feedback the user is can improve its poor responses or minimize issues prompted. Upcoming issue with Augmented Reality system can be deliberate tracking execution or performance. This is for most part innovation based and ideally will be minimized later on. Until this issue is tackled, developers and designers need to consider and attempt to outline the interface in a way that poor tracking execution does not affect more by execution of task $[8,19]$. From the previous research, it is found that accommodating interaction between users is the reason of slow tracking in augmented reality application. From the previous research, a model had been proposed, an answer which adjusts the nature of perception as indicated by the blunder level got from the enrollment. Henceforth, the users have pervasive feedback regarding the system status.

\section{G. Error Tolerance}

Few Augmented reality systems are still in the primary improvement phases and subsequently very inclined to unpredictability. Designers still need to understand issues related to development before such frameworks may truly be mistake tolerant and agree to this design principle. One major difficulty previous described is of tracking Security. Numerous proficient and precise procedures have been created for the top superiority spatial enlistment of actual and simulated info [16]. However arithmetical mistake approximations, natural circumstances (e.g. evolving sunny) or blunders by human brings about errors, for example, virtual info "hopping" all of a sudden vanishing. Newly, proficiently merging dissimilar procedures, having numerous concurrent trackers running in parallel, and recognizing and reunderstanding blunder situations can enhance the influence of the system and consequently decrease user prevention [15].

\section{H. Reducing Cognitive Burden}

To reduce Cognitive burden interface design for the user plays a vital role in performing the real task. VR uses in certain examples may cause the formation of extra features, as a result, increasing in cognitive efforts to use the system thus increasing the distraction for the users and deviating from the main objective of the AR system, for e.g. the AR system with new and unverified interaction representations. The cognitive load for the experts and designer of the specific system tend to be very low but the same can't be said about the novice users, and as a result may be demanding for novice users. According to researchers, cognitive overhead may cause a decrease in learning effects in virtual learning environments. It is thought that if the cognitive load is very huge it may prove AR not as effective as it is considered to be. Renowned Computer scientists like Kaufmann and Schmalstieg lay great emphasizes on the fact the major focused of augmented reality and virtual reality interfaces is to keep the focus of its user on real task rather than making them mastering the interface due to which a lot of mental and physical efforts is being put into the system [17]. The performance of users and the available features have a very strong link, and since the interface acts as a bridge between the user and features, many AR systems encounter the problem that not all features are not tested, several errors like registration error and the use of cognitive skill of the user in understanding objects may hamper the user performance.

\section{Flexibility}

User "Preferences and Abilities" are the major factor while designing and evaluating the design of the augmented reality interface and system or environment, while developing and designing any application user preferences and facilities are two most important aspect of UI, so while designing the augmented reality interface designers should give this aspect more importance. An intriguing feature of AR innovation is the likelihood of incorporating various types of I/O devices. In order to accommodate user preference different modalities should be integrated. To achieve specific task few info modalities are more appreciated, while supporting numerous interaction methods or approaches provides the user with more decision. According to Scott Green "Exceptional modalities can recover each other and the exchange now is among timemultiplexed and space multiplexed devices either hardware or software" [18]. 


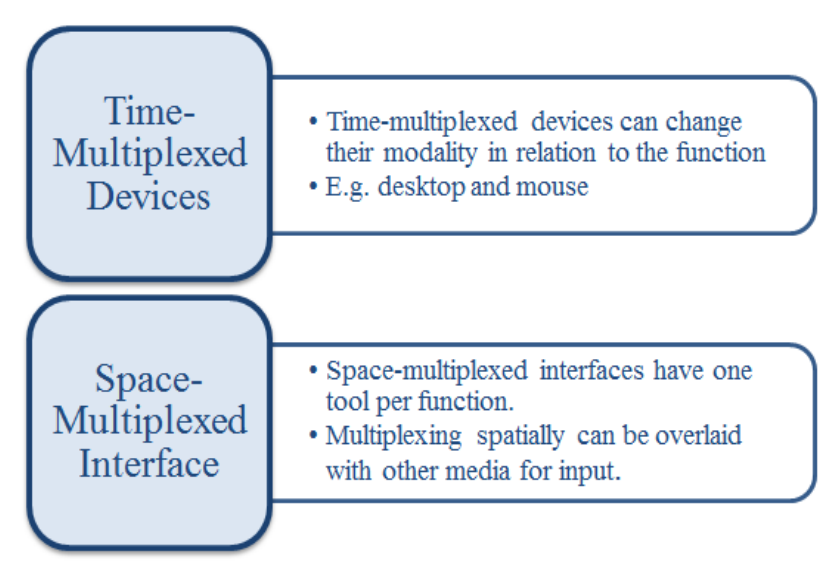

Fig. 3. Classification of Unique Modalities.

Additionally, it also includes Scalability which includes how well the interactions map to different environments which include gestural interaction on object of multiple sizes as shown in Fig. 3.

\section{J. Simplicity}

An efficient interface for the users convey what the user is doing, what user looking for, is there any emergence exit which help user to go back where it come from. Neglecting to convey on any of these guarantees will just prompt to sentiment dissatisfaction or perplexity. You should acknowledge the way that individuals won't utilize your item on the off chance that it is baffling, regardless of what its specialized abilities and determinations are. Here Simplicity and Effortlessness must be an overwhelming need (Ware and Balakrishnan 1994).

More than this an efficient interface must be predictable as well. This means the interface gives users certainty of what will happen when they collaborate with it. Accomplishing this objective requires the steady adherence to basic yet versatile standards and examples all through the interface. Utilize predictable procedures for sorting out, adjusting, and requesting interface components. The final product is a framework that is speedier to learn, less demanding to ace, and results in less oversight [6].

\section{ReSEARCH Methodology}

The review is quantifiable in nature and the tests were carried out in two ways: responses were collected from both novice users and expert users. In order to test our defined factors we selected a game name "Pokémon Go" as its most recent Augmented Reality game. According to Niantic "Pokémon Go is a free-to-play, location-based augmented reality game developed by Niantic for IOS, Android, and Apple Watch devices". We select "Pokémon Go" and based on above factors Affordance, learnability, efficiency, effectiveness, satisfaction, mental efforts, feedback, tolerance, flexibility and simplicity we developed a questionnaire. The questionnaire reviews were developed using Google Forms. The explanation behind making the structures on Google was to encourage the way toward getting reactions from the user (both expert and new or novice). Google Form can be effortlessly gotten to from the Chrome program, which is the most widely recognized program utilized by the greater part of individuals. By making Google Forms, we could get them filled through email by the expert's users. For the tenderfoot users we made the structures accessible through Facebook to the different university student understudies and afterward directed sessions in our supervision were they were made a request to play the amusement and fill the studies.

\section{A. Questioner Development}

To accumulate user criticism about the amusement and to execute client encounter testing we have formulated a few examiners. The examiner is developed correctly and strategically to get the most out of the user tests and get the bits of knowledge that will help enhance the client encounter. For this amusement we have composed examiners remembering learner user thusly the phrasing utilized as a part of the examiner is straightforward we have abstained from utilizing industry languages like 'sub-route' and 'affordance'. The inquiries are shut finished to guarantee precise information investigation so that distinct outcomes can be created which can additionally help enhance the amusement [20].

The inquiries are kept to a base question to maintain a strategic distance from the client getting disappointed while filling the examiner. The scaling framework utilized is the likert scale and the semantic differential scale. Likert scales utilize set decision answer arranges and are intended to evaluate demeanors or conclusions. This scale measures level of assertion and contradiction. Semantic differential scale is utilized to quantify the demonstrative importance of things or ideas.

\section{Participants}

\section{B. Sampling Technique}

While it is hard to get reactions from an entire populace, inspecting is an endeavor to reach an inference in light of a little representation in a given populace. For my approach I pick arbitrary examining the motivation behind picking irregular inspecting technique is that it needs just a base learning of the review gathering of populace ahead of time, it is free from blunders in characterization, it is reasonable for information investigation which incorporates the utilization of inferential insights. Straightforward arbitrary inspecting is illustrative of the populace and it is thoroughly free from inclination and bias. In this review there were 66 arbitrary users. They users ought to utilize PDAs and have commonality with playing recreations on a touch screen $[18,20]$. The members were told to introduce the amusement on their advanced mobile phones and after that as needs be partake in the review. The members were made a request to give criticism on the ease of use and adequacy of the amusement's interface. The members were advised before they took the study to give legitimate input and consequently overviews were filled by just those users who enthusiastically volunteered to take care of out the surveys with a specific end goal to gather perfect and important information. 


\section{Ethics}

All participants involved were strictly required to follow the following ethical guidelines [19]:

- Participants were to round out the survey forms with trustworthiness and simply after they have introduced and played the amusement themselves.

- Participants were required to fill out the form separately and were made a request to give their name and right age.

- Participants were altogether advised about the reason for the overview with the goal that they could make an educated judgment about whether they need to take an interest in the survey or not.

- Volunteer based participation in the study

- Privacy regarding the response were guarantee to participants

\section{Procedure of Data Collection and Method of Analyzing}

While making the question there were five factors which is keep in mind that is related Affordance, learnability, efficiency, effectiveness, satisfaction, mental efforts, feedback, tolerance, flexibility and simplicity. Learnability, Efficiency Simplicity focus on the areas related to task success, user satisfaction and tolerance. While effectiveness is covering the factors related to time on task, mental effort and memorability covering the factor related to playability of the game, usage of the game and no of errors. The method used for analyzing of result is based on the graphs which we get Google forms. Based on these analyses we conclude our suggested factors are the major factors while developing any augmented reality application

\section{RESUlT AND FINDING}

In below figures are some of the results from the game Pokémon Go related to Factors which are listed above:

The layout lucid and conformed to various resolutions.
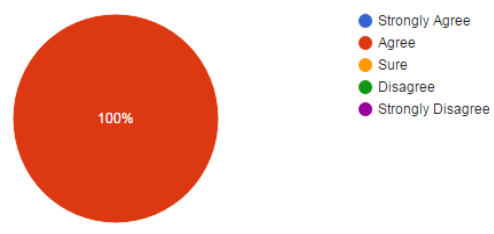

It is consistent.
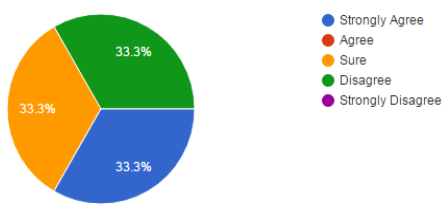

Fig. 4. Result of Layout and Consistency of Game.
From the above analysis shown in Fig. 4 it is clearly shown that experts user feels the layout of the application is good as the layout conformed to various resolutions and. 33.3 $\%$ of the user find the application consistent as the layout used is similar to the user and user easily understand the metaphors used in the game.

From the above analysis shown in Fig. 5 it is clearly shown that $33.3 \%$ expert user feel the color scheme used is average means it is violating rules describe by the color scheme, $66.7 \%$ user feel that it supports task implementation, while 33.3 feels that it doesn't support the task implementation due its consistency issue.

From the above analysis shown in Fig. 6 it is clearly shown due to issues in the color scheme the complexity of the application is legitimate. While $33.3 \%$ of the expert that due to complexity issues simplicity issues arises in the application. But $33.3 \%$ experts said the overall Simplicity of the application was average due to natural mapping of the virtual object with real world object.

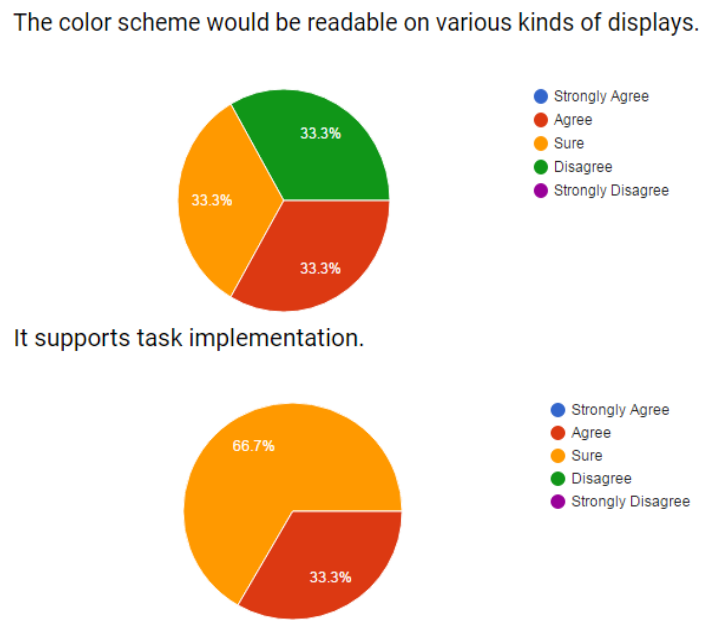

Fig. 5. Result of Color Scheme on Various Display and Task Implementation.

There is legitimate complexity amongst text and background.
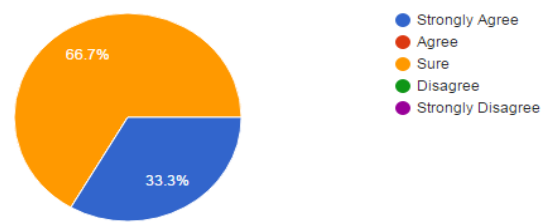

The access to all elements of an application simple and instinctive.
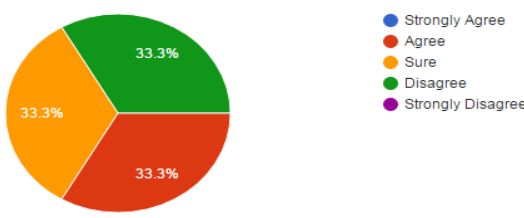

Fig. 6. Result of Complexity and Simplicity. 
The data show up in places, where it is required.

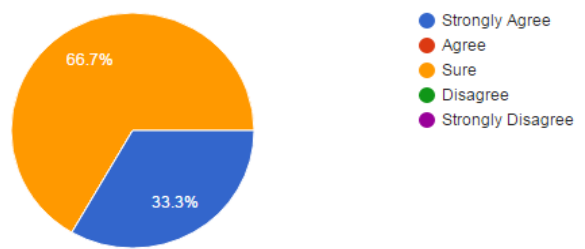

They give enough data on the status of activities performed by user.

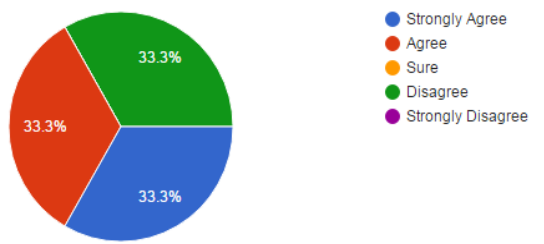

Fig. 7. Result of Complexity and Simplicity.

From the above analysis shown in Fig. 7 a conclusion is made expert find the placement of the object satisfactory as most of the object are not placed or map according to the realworld object there are some unnecessary features are adding on the game, the design is not minimalistic.

The interface naming is easy to understand for it's targeted users.

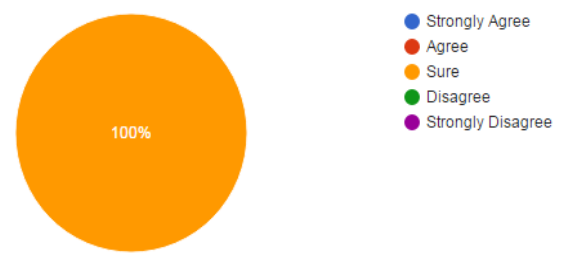

The storyboard is predictable and very much arranged.

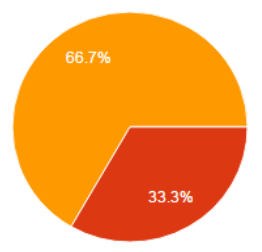

- Strongly Agree

- Agree

Sure

- Disagree

- Strongly Disagree

Fig. 8. Result of Predictability and Understandability.

From the above analysis shown in Fig. 8 conclusion is made expert find the predictability of the elements in the game is satisfactory as most of the object cannot recognized by the user as the mapping of the object are not good while few users think it's perfect. While majority of the user understand the game easy but still they some kind of help to play the game as the learnability of game is satisfactory.

From the above analysis shown in Fig. 9, conclusion is made expert find the simplicity of the game is satisfactory as the game need more enhancement, this problem occurs due lack of help and document there are very less hints provided for user in game.
The access to all segments of an application simple and instinctive.

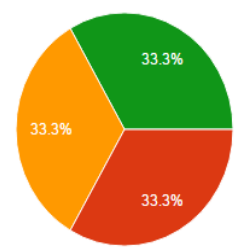

- Strongly Agree

Sure

- Disagree

- Strongly Disagree

They contain hints on occurring problems

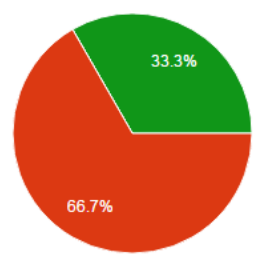

- Strongly Agree

- Agree

Sure

Disagre

- Strongly Disagree

Fig. 9. Result of Help and Documentation and Simplicity of Segments in Game.

After concluding all the above analysis we come to a conclusion that these principles are most needed and important while developing any AR application or system as they play greater role in making the usability of the interface easier for the users and make it interactive.

\section{CONCLUSION}

Our Research proposed few well know GUI design principles for augmented reality system and interfaces with help which AR system and interfaces become more interactive and easily understandable for the user as they identify major issues which user face while interacting with AR systems and interfaces. Since this is initial stage to overcome the gap in the augmented reality interfaces in the particular domain. The suggested design principles and guidelines are the small the synopsis and it can be further developed. Since it is very difficult task to generate or suggest the principles which help in improving the augmented reality system. Since selecting and suggestion of specific rules or principles are difficult to process as the current implementation of augmented reality system and input and output devices are pretty miscellaneous. Therefore, it is significant to incorporate study from dissimilar spaces in order to define augmented reality design principles. Our study also proposed different deign principles which help in designing AR system and interfaces. To validate and check whether these principles are helpful or not we apply these principles on Augmented Reality game name Pokémon Go. And from out result we validate that these principles are most important while developing any AR system or interface

\section{REFERENCES}

[1] Gabbard, Joe L, and J Edward Swan II. 2008. "Usability engineering for augmented reality: Employing user-based studies to inform design." IEEE Transactions on visualization and computer graphics 14 (3):513525.

[2] Gabbard, Joseph L, Deborah Hix, and J Edward Swan. 1999. "Usercentered design and evaluation of virtual environments." IEEE computer Graphics and Applications 19 (6):51-59. 
[3] Moore, Antoni. 2006. A tangible augmented reality interface to tiled street maps and its usability testing: Springer.

[4] Nielsen, Jakob, and Rolf Molich. 1990. "Heuristic evaluation of user interfaces." Proceedings of the SIGCHI conference on Human factors in computing systems.

[5] Bowman, Doug A, Joseph L Gabbard, and Deborah Hix. 2002. "A survey of usability evaluation in virtual environments: classification and comparison of methods." Presence: Teleoperators and Virtual Environments 11 (4):404-424.

[6] Stedmon, Alex W, and Robert J Stone. 2001. "Re-viewing reality: human factors of synthetic training environments." International Journal of Human-Computer Studies 55 (4):675-698.

[7] Swan, J Edward, and Joseph L Gabbard. 2005. "Survey of user-based experimentation in augmented reality." Proceedings of 1 st International Conference on Virtual Reality.

[8] Ware, Colin, and Ravin Balakrishnan. 1994. "Reaching for objects in VR displays: lag and framerate." ACM Transactions on ComputerHuman Interaction (TOCHI) 1 (4):331-356.

[9] Coelho, Enylton Machado, Blair MacIntyre, and Simon J Julier. 2004 "OSGAR: A scene graph with uncertain transformations." Proceedings of the 3rd IEEE/ACM International Symposium on Mixed and Augmented Reality.

[10] Rizzo, Albert A, Gerard J Kim, Shih-Ching Yeh, Marcus Thiebaux, Jayne Hwang, and J Galen Buckwalter. 2005. "Development of a benchmarking scenario for testing 3D user interface devices and interaction methods." Proceedings of the 11th International Conference on Human Computer Interaction, Las Vegas, Nevada, USA.

[11] Billinghurst, Mark, Hirokazu Kato, and Ivan Poupyrev. 2001. "The MagicBook: a transitional AR interface." Computers \& Graphics 25 (5):745-753.

[12] Bowman, Doug A, Joseph L Gabbard, and Deborah Hix. 2002. "A survey of usability evaluation in virtual environments: classification and comparison of methods." Presence: Teleoperators and Virtual Environments 11 (4):404-424.

[13] Broll, Wolfgang, Irma Lindt, Jan Ohlenburg, Iris Herbst, Michael Wittkamper, and Thomas Novotny. 2005. "An infrastructure for realizing custom-tailored augmented reality user interfaces." IEEE transactions on visualization and computer graphics 11 (6):722-733.

[14] Ware, Colin, and Ravin Balakrishnan. 1994. "Reaching for objects in VR displays: lag and framerate." ACM Transactions on ComputerHuman Interaction (TOCHI) 1 (4):331-356.

[15] Coelho, Enylton Machado, Blair MacIntyre, and Simon J Julier. 2004. "OSGAR: A scene graph with uncertain transformations." Proceedings of the 3rd IEEE/ACM International Symposium on Mixed and Augmented Reality.

[16] Avery, Benjamin, Wayne Piekarski, James Warren, and Bruce $\mathrm{H}$ Thomas. 2006. "Evaluation of user satisfaction and learnability for outdoor augmented reality gaming." Proceedings of the 7th Australasian User interface conference-Volume 50.

[17] Stone, Debbie, Caroline Jarrett, Mark Woodroffe, and Shailey Minocha. 2005. User interface design and evaluation: Morgan Kaufmann.

[18] Irawati, Sylvia, Scott Green, Mark Billinghurst, Andreas Duenser, and Heedong Ko. 2006. "Move the couch where?: Developing an augmented reality multimodal interface." Proceedings of the 5th IEEE and ACM International Symposium on Mixed and Augmented Reality.

[19] Ware, Colin, and Ravin Balakrishnan. 1994. "Reaching for objects in VR displays: lag and rate." ACM Transactions on Computer-Human Interaction (TOCHI) 1 (4):331-356.

[20] Wharton, Cathleen, John Rieman, Clayton Lewis, and Peter Polson. 1994. "The cognitive walkthrough method: A practitioner's guide." Usability inspection methods. 